\title{
Updates on metaQuantome software for quantitative metaproteomics
}

Subina Mehta ${ }^{1}$, Praveen Kumar ${ }^{1}$, Marie Crane ${ }^{1}$, James. E Johnson², Ray Sajulga1, Dinh Duy An Nguyen $^{1}$, Thomas McGowan ${ }^{2}$, Magnus $\varnothing$. Arntzen ${ }^{3}$, Timothy. J Griffin ${ }^{1}$ and Pratik. D Jagtap ${ }^{1^{*}}$

${ }^{1}$ Department of Biochemistry, Molecular Biology, and Biophysics, University of Minnesota Twin Cities, Minneapolis USA

${ }^{2}$ Minnesota Supercomputing Institute, University of Minnesota Twin Cities, Minneapolis USA

${ }^{3}$ Faculty of Chemistry, Biotechnology and Food Science, NMBU, Ås, Norway

* Corresponding author: Pratik Jagtap (pjagtap@umn.edu)

Supporting Information:

Figure S1: Three metaQuantome bar plot outputs showing the top five GO molecular functions along with its peptide abundance

Figure S2: The metaQuantome visualization module generates four outputs for the taxonomy mode.

Figure S3: metaQuantome visualization tools generate different outputs from the MT2MQ metatranscriptomics data. 
$8 \mathrm{hr}(\mathrm{T} 2)$

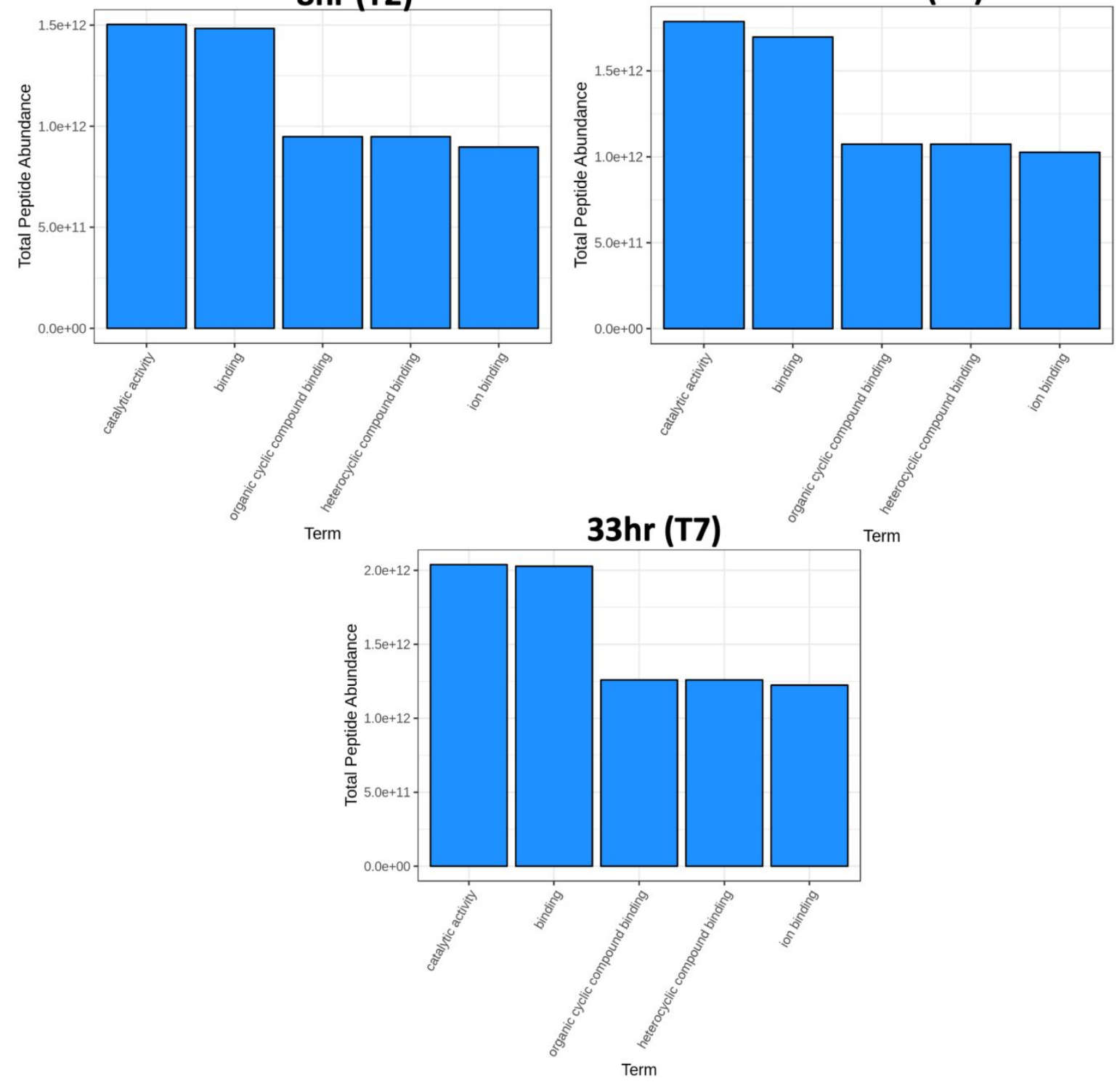

Figure S1: Three metaQuantome bar plot outputs showing the top five GO molecular functions along with its peptide abundance expressed in $8 \mathrm{hr}$ (T2), $18 \mathrm{hr}$ (T4) and 33hr (T7) timepoints. 

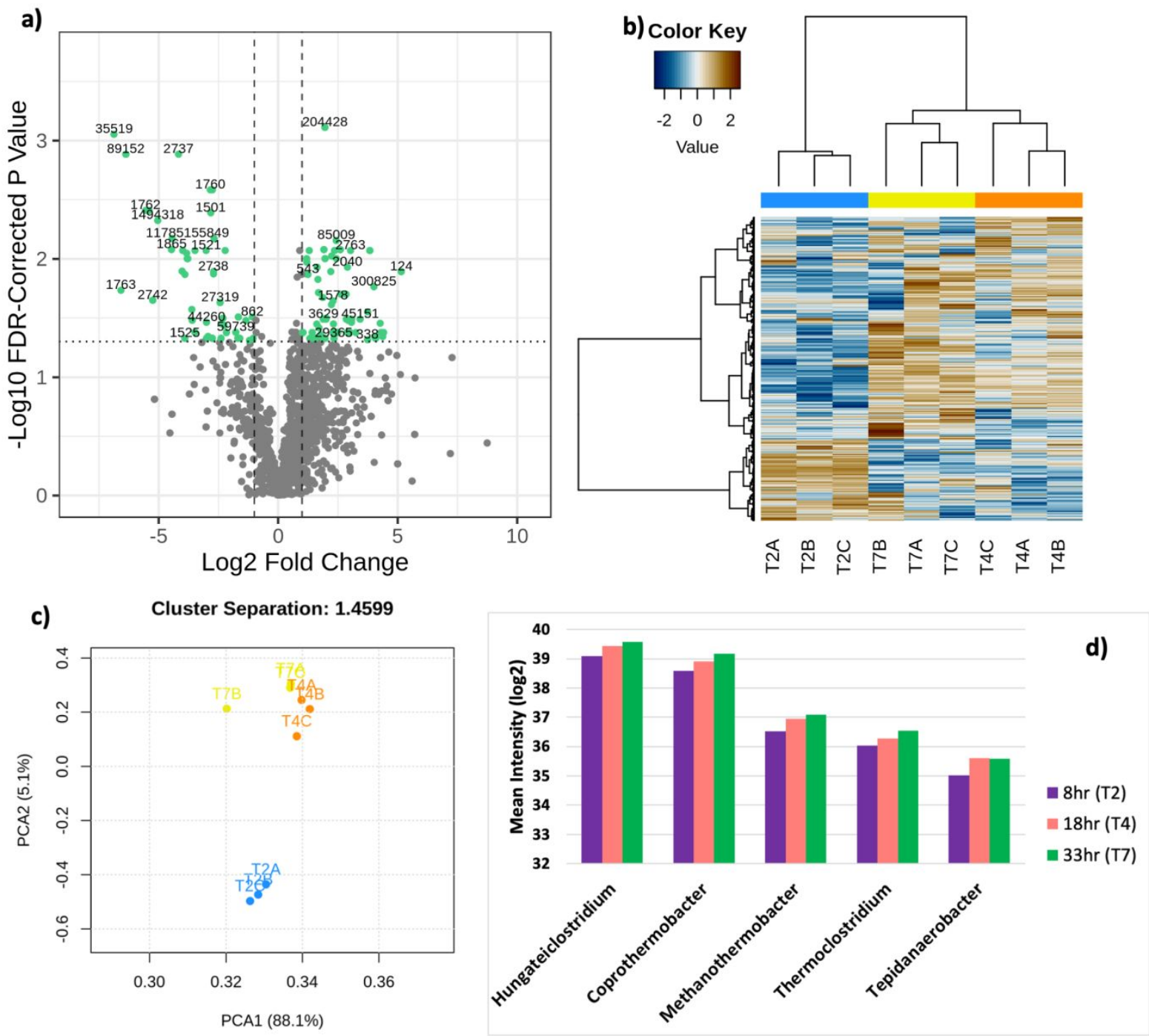

Figure S2: metaQuantome taxonomic outputs: The metaQuantome visualization module generates four outputs for taxonomy mode: a) Volcano plot shows various differentially expressed taxa between $33 \mathrm{hr}$ (T7) and $8 \mathrm{hr}$ (T2) time. b) Heatmap visualization comparing the differentially expressed taxonomic units in each dataset. c) PCA plot represents the cluster separation between each of the time points and its replicates. d) Bar plot generated using stat data for the five top most abundant genera identified. 

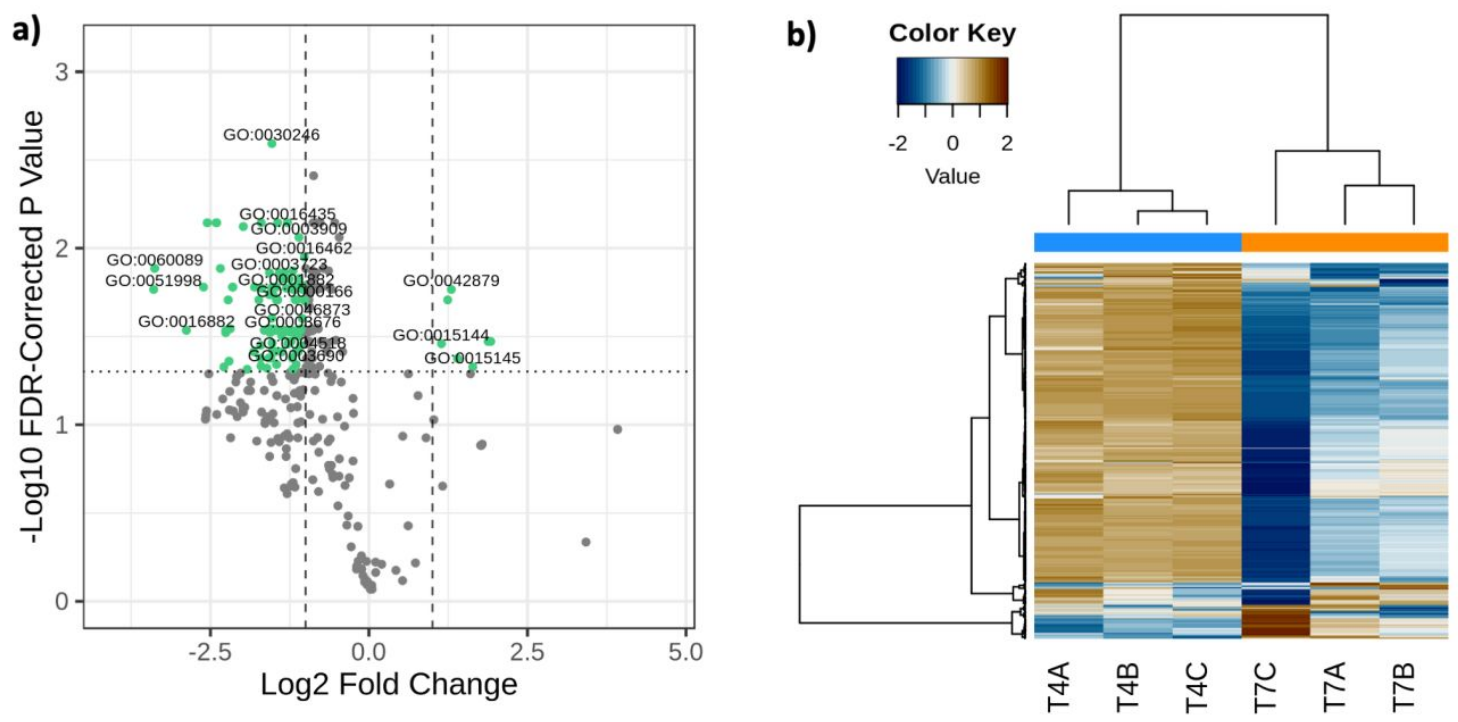

c) Cluster Separation: $\mathbf{2 . 8 2 1 5}$
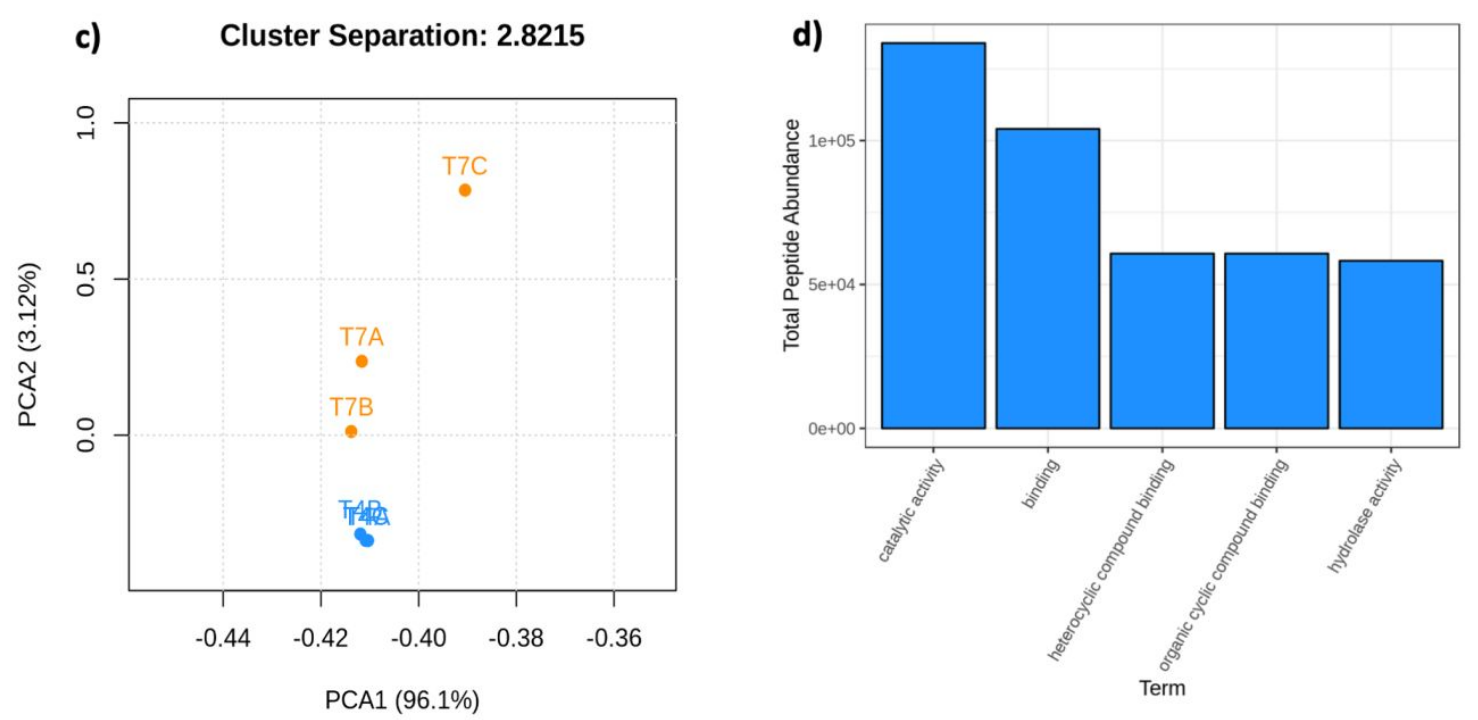

Figure S3: metaQuantome output on MT2MQ inputs: metaQuantome visualization tools generates four outputs for the metatranscriptomics data: a) Volcano plot comparing $18 \mathrm{hr}$ (T4) and 33hr (T7) shows various differentially expressed GO Terms $b$ ) heatmap showing level of expression between the two datasets and also denotes that same time points were clustered together c) PCA plot describes the cluster separation between the samples d) Bar plot representing the five topmost GO molecular functions expressed in at time point $18 \mathrm{hr}$ (T4). 